\title{
Macromoluscos intermareales de sustratos rocosos de la playa Buque Quemado, Estrecho de Magallanes, sur de Chile
}

Intertidal macromolluscs from the rocky substrata of Buque Quemado Beach, Strait of Magellan, southern Chile

\section{Cristian Aldea ${ }^{1}$ y Sebastián Rosenfeld ${ }^{2}$}

\begin{abstract}
${ }^{1}$ Fundación Centro de Estudios del Cuaternario de Fuego-Patagonia y Antártica (CEQUA), Universidad de Magallanes, Av. Bulnes 01890, Casilla 737, Punta Arenas, Chile. cristian.aldea@cequa.cl

${ }^{2}$ Departamento de Ciencias y Recursos Naturales, Facultad de Ciencias, Universidad de Magallanes, Avenida Bulnes 01855, Casilla 113-D, Punta Arenas, Chile. srosenfe@umag.cl.
\end{abstract}

\begin{abstract}
The Strait of Magellan is a complex natural channel that connects the Pacific and Atlantic Oceans. Due to the weather and oceanographic characteristics of this zone, scientists have been interested in its biota, generating numerous expeditions and studies focused to the marine ecosystems. However, maj or research has been oriented towards sublitoral environments, creating a lack in the knowledge of numerous intertidal places. The Buque Quemado Beach, located on the oriental end of the Strait of Magellan, corresponds to an area dominated by soft substrata, and it has not yet been characterized from the malacological point of view. In April 2008, exhaustive sampling using a transect perpendicular to the coast line, from the upper intertidal to the lower intertidal was carried out, where all molluscs were collected, which were then fixed, identified and photographed; carrying out observations on the taxonomy, ecology, distribution and biogeography of all species. Twelve species were identified ( 2 Polyplacophora, 9 Gastropoda and 1 Bivalvia) from the 218 examined specimens, of which $69 \%$ corresponded the bivalve Mytilus edulis chilensis. Fifty eight percent of the species exhibited a Magellan distribution and the $42 \%$ remaining demonstrated a wide distribution embracing two or more biogeographic provinces. The low number of obtained species could respond mainly to physical factors of the beach, considering that soft substrates act as an important spatial factor, shrinking intertidal diversity.
\end{abstract}

Key words: Taxonomy, ecology, biogeography, Polyplacophora, Gastropoda, Bivalvia

Resumen. - El Estrecho de Magallanes corresponde a un complejo canal natural de conexión entre los océanos Pacífico y Atlántico. Debido al clima y características oceanográficas de esta zona, los científicos se han interesado por su biota, generando numerosas expediciones y estudios enfocados al ecosistema marino. Sin embargo, muchas de las investigaciones realizadas se han orientado a ambientes sublitorales, existiendo una carencia en el conocimiento de numerosos sitios intermareales. La playa Buque Quemado, ubicada en el extremo oriental del Estrecho de Magallanes, corresponde a un área dominada por sustratos blandos, no caracterizada desde el punto de vista malacológico. En abril de 2008 se realizó un muestreo exhaustivo de un transecto perpendicular a la línea de costa desde la zona del intermareal superior hasta el intermareal inferior, colectándose todos los moluscos encontrados, los cuales fueron fijados, identificados y fotografiados; realizando observaciones sobre la taxonomía, ecología, distribución y biogeografía de todas las especies. Se determinaron 12 especies (2 Polyplacophora, 9 Gastropoda y 1 Bivalvia) a partir de 218 especímenes examinados, de los cuales el $69 \%$ correspondió al bivalvo Mytilus edulis chilensis. El 58\% de las especies son propias de la provincia Magallánica y el $42 \%$ restante incluye especies que se distribuyen en dos o más provincias biogeográficas. El bajo número de especies obtenidas podría responder mayormente a factores físicos de la playa, sugiriendo considerar los sustratos blandos como un importante factor espacial limitante de la diversidad intermareal.

Palabras clave: Taxonomía, ecología, biogeografía, Polyplacophora, Gastropoda, Bivalvia

\section{INTRODUCCIÓN}

La Región de Magallanes se caracteriza por presentar un particular sistema de fiordos y canales. Uno de los más importantes es el Estrecho de Magallanes, que corresponde a una de las vías naturales del mundo que conecta dos océanos, el Pacífico y el Atlántico. Entre las características batimétricas más notables, el Estrecho de Magallanes puede 
ser dividido en tres micro-cuencas: la oriental, ubicada entre la entrada del Océano Atlántico y la Segunda Angostura, la central desde Segunda Angostura hasta la Isla Carlos III, y la occidental desde la Isla Carlos III hasta la entrada del Océano Pacífico (Valdenegro \& Silva 2003). Debido a estas características, la biota marina de la zona de Magallanes es representativa por su alta diversidad y heterogeneidad en tipos de hábitat marino-costeros, que sustentan una gran diversidad de algas e invertebrados (Camus 2001).

Gran parte del conocimiento sobre los moluscos en la Región de Magallanes se debe a las numerosas expediciones científicas realizadas desde el siglo XIX. A comienzos del siglo XX se realizó una importante campaña de investigación Alemana y Sueca en la región magallánica (Strebel 1904, 1905 a, b, 1906, 1907) y años más tarde, entre 1925-1951, se llevó a cabo la campaña Discovery (Powell 1951, Dell 1964) con el fin de investigar y obtener mayor conocimiento sobre el Océano Austral. Otras expediciones que aportaron enormemente al conocimiento de la fauna de los fiordos entre Puerto Montt y el Estrecho de Magallanes fueron la expedición de la Universidad de Lund entre 1948-1949 (e.g., Marcus 1959, Soot-Ryen 1959) y la expedición de la Royal Society entre 1958-1959 (Dell 1971). Entre las expediciones más actuales se encuentran la ‘Victor Hensen’ en 1994 (e.g., Linse 1997) y los cruceros CIMAR-FIORDOS II y III entre 1996-1998 (Palma \& Silva 2006). Todas estas investigaciones generaron un gran aporte científico en diversas áreas de las ciencias del mar y ayudaron a tener un mejor conocimiento sobre la Región de Magallanes.

No obstante a lo anterior, falta conocimiento de diversos puntos intermareales de los canales y áreas de fiordos, entre ellos, de sitios localizados en el Estrecho de Magallanes, como uno de los canales más importantes en la región. Trabajos modernos reportando moluscos intermareales son aún escasos (e.g., Mutschke et al. 1998), contabilizándose mayormente trabajos comunitarios en la micro-cuenca central, enfocados al área entre Bahía Laredo y Bahía Mansa en la Península de Brunswick (e.g., Benedetti-Cecchi \& Cinelli 1997, Ríos \& Gerdes 1997) y al Canal Whiteside (Ríos \& Mutschke 1999). Muchos de los sitios aún no caracterizados se localizan en áreas de influencia humana a través de actividades marítimas, portuarias, pesqueras o urbanas. El objetivo de este trabajo fué describir la macrofauna de moluscos presentes en los sustratos rocosos de la playa Buque Quemado del área denominada Primera Angostura, en la micro-cuenca oriental del Estrecho de Magallanes.

\section{Materiales y Métodos}

El área de estudio comprende la playa Buque Quemado, ubicada geográficamente en el lado oriental del Estrecho de Magallanes frente a la Isla de Tierra del Fuego (52²8’S;

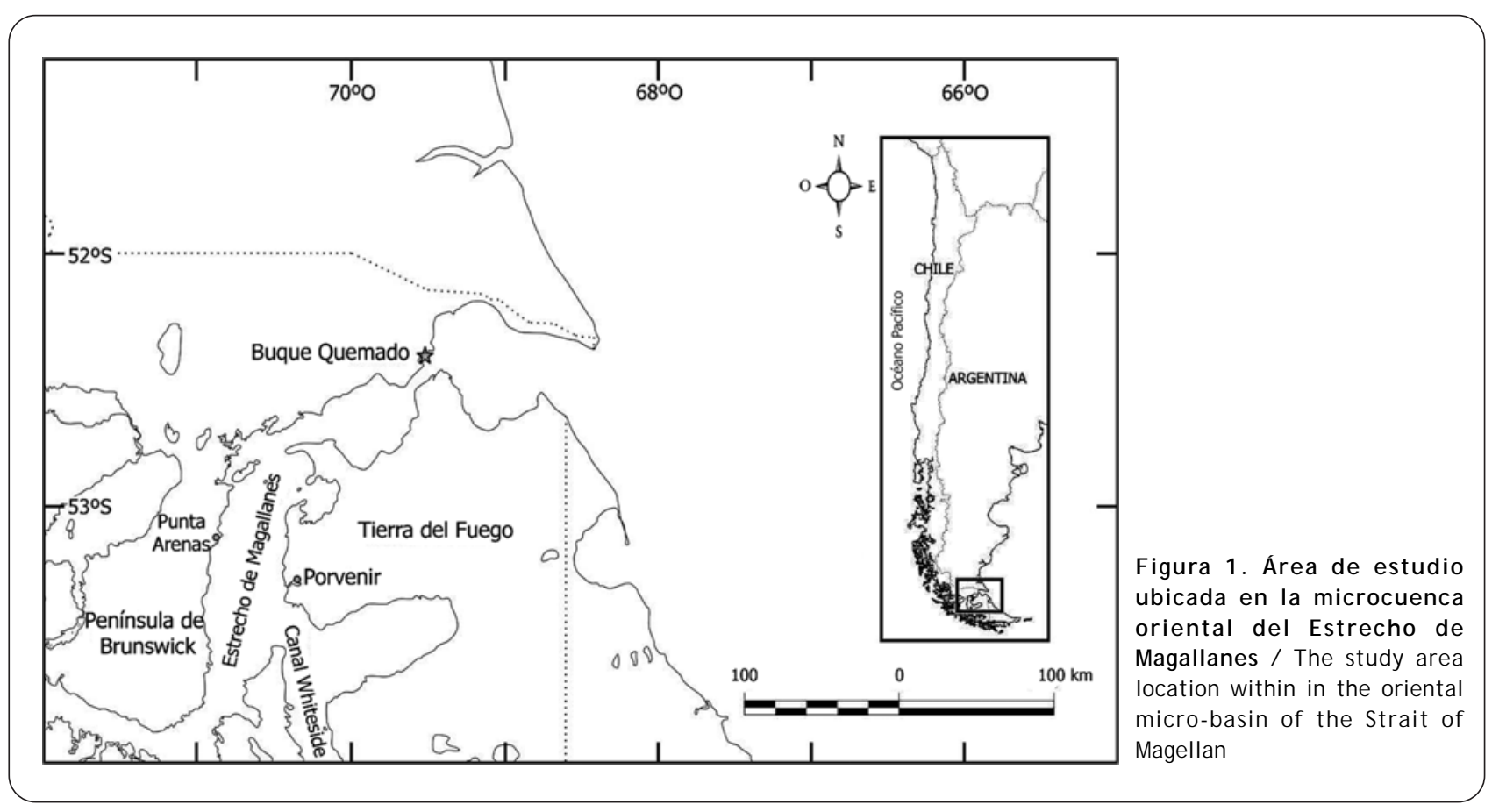


69³3’O) (Fig. 1). El sector, al pertenecer a la zona llamada micro-cuenca oriental del Estrecho de Magallanes, está influenciado principalmente por aguas provenientes del océano Atlántico. El substrato de esta playa está compuesto mayormente de fondos blandos con parches de roca o bolones pequeños, cuya zona submareal también se caracteriza por presentar bosques de Macrocystis pyrifera, los cuales constituyen un importante sustento para los ecosistemas bentónicos. Una característica importante que presenta esta playa es su gran amplitud de marea, la que puede llegar a descender más de $3 \mathrm{~km}$.

El estudio se realizó en abril de 2008. Durante marea baja se efectuó una exhaustiva inspección visual en un transecto perpendicular a la línea de costa desde la zona del intermareal superior hasta el intermareal inferior, realizando una colecta in situ de organismos vivos mediante extracción manual y espátulas. Los moluscos recolectados fueron inmediatamente puestos en bolsas plásticas rotuladas y conservados en alcohol al 96\%. Posteriormente los ejemplares fueron llevados al laboratorio, donde fueron revisados, identificados y fotografiados en un estereomicroscopio Olympus SZ61 adosado a una cámara Moticam 2000; luego fueron medidos utilizando un calibre digital.

Para la identificación y la caracterización de las especies (i.e., distribución, observaciones taxonómicas y ecológicas) se utilizó como referencia toda la literatura disponible para los moluscos de la región magallánica y áreas vecinas, incluyendo trabajos de revisiones a taxones específicos (e.g., Zelaya 2004). En el ámbito biogeográfico, siguiendo la clasificación de ‘provincias’ biogeográficas propuestas por
Stuardo (1964) para los moluscos litorales de Latinoamérica, la descripción zoogeográfica de Brattström \& Johanssen (1983) y la revisión de Camus (2001) para la costa chilena, se definió la distribución de las especies como 'magallánicas' y de amplia distribución 'peruana-magallánica', ‘magallánica-patagónica’ y ‘peruano-magallánicapatagónica’.

\section{RESUltados}

De un total de 218 moluscos recolectados (Tabla 1), se determinaron 12 especies, correspondientes a 10 familias. En términos de riqueza por clases, Gastropoda estuvo representada por 9 especies, Poplyplacophora por dos y Bivalvia sólo por una especie. En términos de abundancia, la clase con mayor número de individuos fue Bivalvia ( $n=151$ ), representando el $69 \%$ de los moluscos colectados que correspondían a la especie Mytilus edulis chilensis.

Desde el punto de vista biogeográfico (Fig. 2), el 58\% de las especies exhibió una distribución magallánica: Nacella deaurata, Pareuthria plumbea, Kerguelenella lateralis, Tonicia lebruni, Trochita pileus, Fissurella picta picta y Plaxiphora aurata; las últimas tres se distribuyen por el norte hasta el área intermedia entre las provincias Magallánica y Peruana. El 34\% demostró una amplia distribución 'peruana-magallánica', Scurria ceciliana y Mytilus edulis chilensis y 'magallánica-patagónica', Nacella magellanica y Margarella expansa. Mientras que la especie Siphonaria lessoni, el 8\% del total, mostró una amplia distribución que comprendió las tres provincias biogeográficas.

Tabla 1. Moluscos intermareales recolectados en la playa Buque Quemado, Estrecho de Magallanes / Intertidal molluscs collected within Buque Quemado Beach, Strait of Magellan, Chile

\begin{tabular}{lllr}
\hline Clase & \multicolumn{1}{c}{ Familia } & \multicolumn{1}{c}{ Especie } & Individuos \\
\hline \multirow{2}{*}{ Polyplacophora } & Chitonidae & Tonicia lebruni Rochebrune, 1884 & 3 \\
Gastropoda & Mopaliidae & Plaxiphora aurata (Spalowsky, 1795) & 3 \\
& Fissurellidae & Fissurella picta picta (Gmelin, 1791) & 9 \\
& Nacellidae & Nacella deaurata (Gmelin, 1791) & 11 \\
& & Nacella magellanica (Gmelin, 1791) & 23 \\
& Lottiidae & Scurria ceciliana (Orbignyi, 1841) & 1 \\
& Trochidae & Margarella expansa (Sowerby, 1838) & 12 \\
& Calyptraeidae & Trochita pileus (Lamarck, 1822) & 1 \\
& Buccinidae & Pareuthria plumbea (Philippi, 1844) & 2 \\
Sivhalvia & Siphariidae & Kerguelenella lateralis (Gould, 1846) & 1 \\
& Mytilidae & Siphonaria lessoni Blainville, 1824 & 1 \\
& & Mytilus edulis chilensis Hupé, 1854 & 151 \\
\hline
\end{tabular}




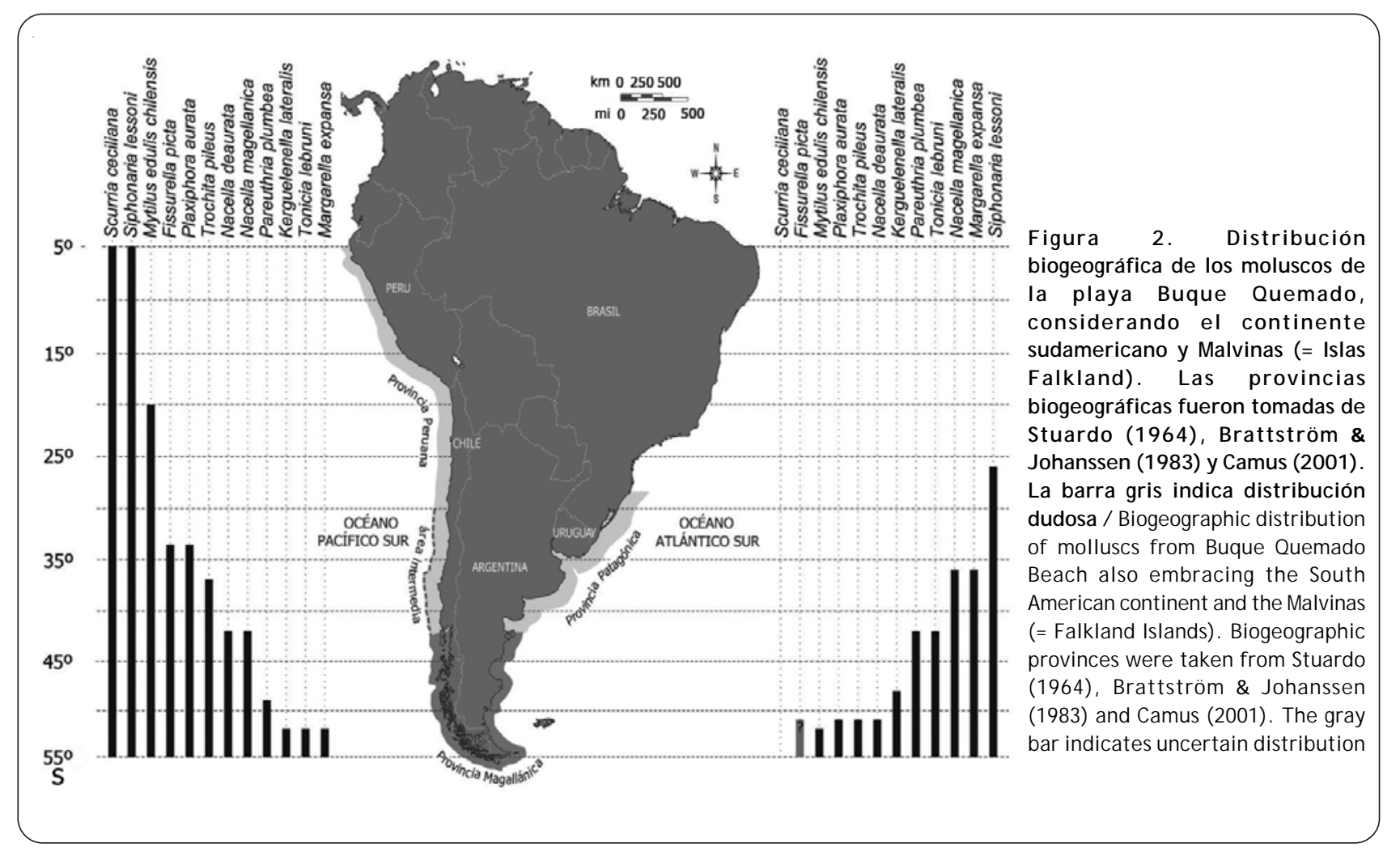

\section{Discusión}

La especie Tonicia lebruni (Fig. 3A-B) se distribuye en el Estrecho de Magallanes hasta Isla de los Estados, extendiéndose hacia Chubut en el Océano Atlántico, con una batimetría desde la zona intermareal a $30 \mathrm{~m}$ de profundidad, siendo además señalado como el poliplacóforo más abundante en aguas someras en el Estrecho de Magallanes e Islas Malvinas/Falkland (Sirenko 2006). Recientemente aún era considerado sinónimo sénior de la especie T. calbucensis Plate, 1898, pero Sirenko (2006) señala que son especies diferentes. Luego $T$. calbucensis es restablecida como especie válida, puntualmente en seis caracteres diferenciales entre ambas especies (ver Schwabe et al. 2006, p. 15).

Plaxiphora aurata (Fig. 3C) es una especie común y conocida que además presenta bastante variabilidad en el color y escultura de las placas (ver Sirenko 2006). Se distribuye entre Valparaiso y el Estrecho de Magallanes, extendiéndose hacia diversas islas subantárticas (ver Schwabe et al. 2006) en una batimetría entre 0 y $25 \mathrm{~m}$ (Sirenko 2006). Reid \& Osorio (2000) mencionan la ocurrencia de la especie en playas fangosas y su tolerancia a bajas salinidades. Su anatomía externa fue redescrita por Schwabe et al. (2006).
El gasterópodo Fissurella picta picta (Fig. 4A-B) es similar en aspecto general a Fissurella radiosa radiosa Lesson, 1831 y Fissurella oriens oriens Sowerby, 1833 (ver McLean, 1984, p. 40, 44, 48), compartiendo puntos de distribución geográfica. Este mismo autor además estudió la morfología, distribución y sistemática de las distintas especies del género presentes en el Pacífico sur-oriental, reconociendo en este caso dos subespecies geográficas: $F$. picta picta (Gmelin, 1791) y F. picta lata Sowerby, 1835, esta última de distribución más septentrional. F. picta picta es caracterizada como una subespecie abundante de distribución intermareal entre $33^{\circ} \mathrm{S}$ y $56^{\circ} \mathrm{S}$ (Reid \& Osorio 2000), extendiéndose probablemente hasta las Islas Malvinas / Falkland (McLean 1984).

Nacella deaurata (Fig. 4C-D) y N. magellanica (Fig. 4E-G) son especies de nacélidos intermareales característicos en el Estrecho de Magallanes. Powell (1973) publicó la revisión más importante sobre el grupo, considerando a $N$. delicatissima Strebel, 1907 como un ecotipo de $N$. deaurata, y $N$. venosa (Reeve, 1854) y $N$. chiloensis (Reeve, 1855) como subespecies de $N$. magellanica. Años más tarde, Valdovinos \& Rüth (2005) estudiaron la variación morfológica de las distintas especies 


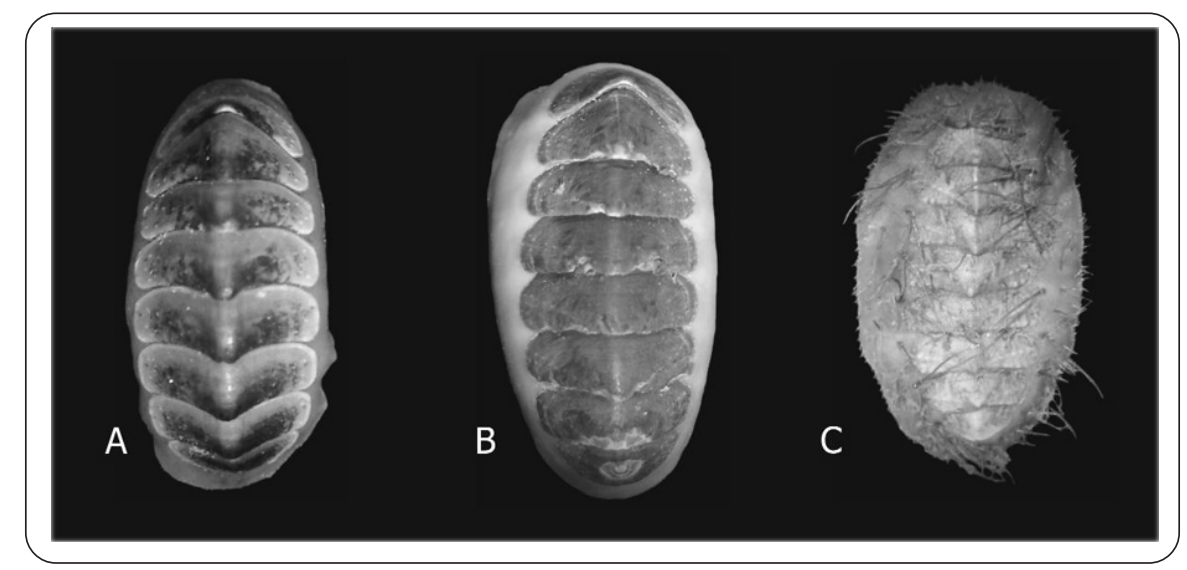

Figura 3. Polyplacophora de la playa Buque Quemado. A) Tonicia lebruni $(8,64 \mathrm{~mm})$, B) Tonicia lebruni $(20,71 \mathrm{~mm})$, C) Plaxiphora aurata $(13,26 \mathrm{~mm})$ / Polyplacophora from Buque Quemado Beach. A) Tonicia lebruni (8.64 mm), B) Tonicia lebruni (20.71 mm), C) Plaxiphora aurata $(13.26 \mathrm{~mm})$

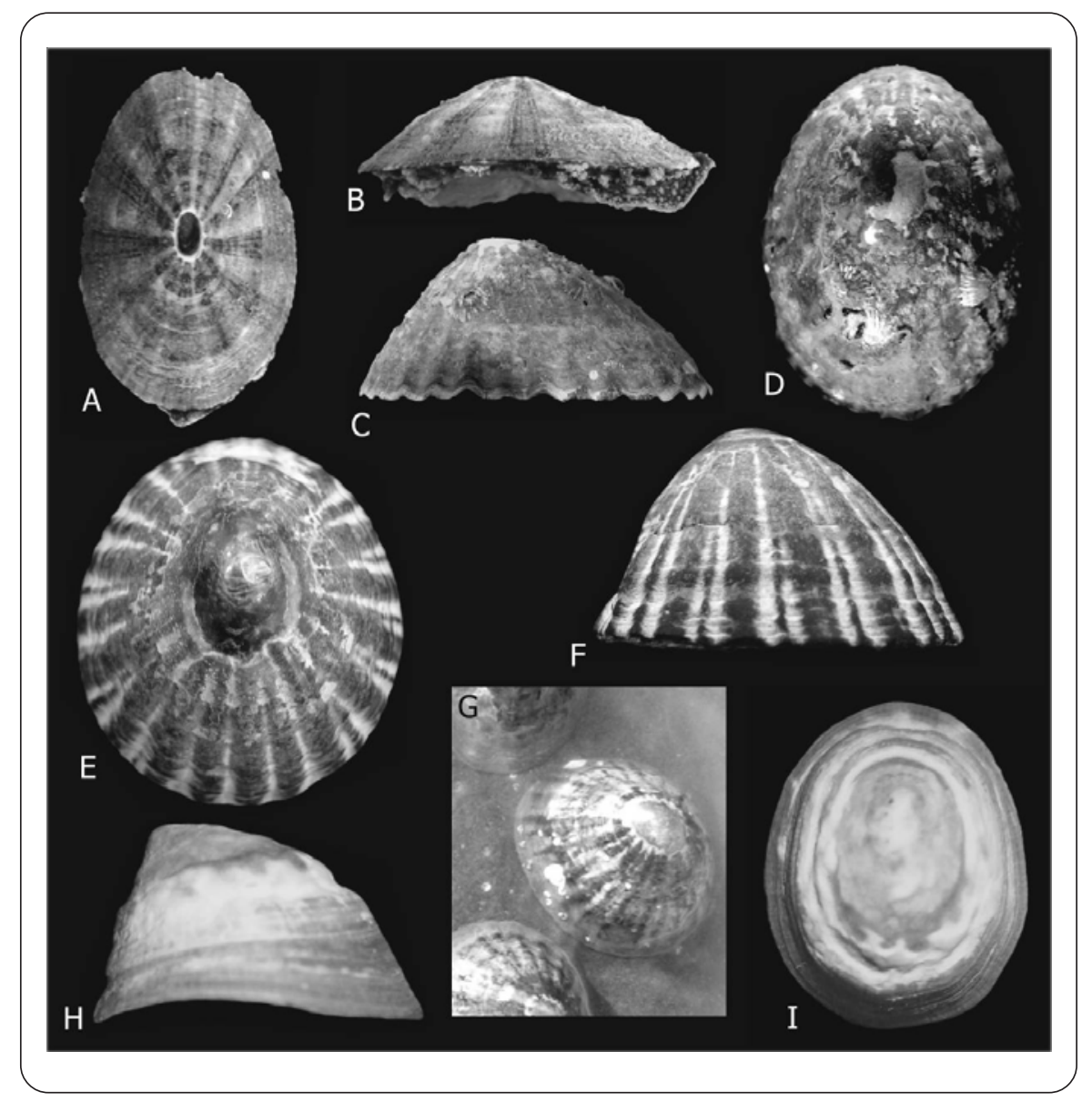

Figura 4. Gastropoda de la playa Buque Quemado. A-B) Fissurella picta picta (50,63 mm), C-D) Nacella deaurata $(42,13 \mathrm{~mm}), \mathrm{E}-\mathrm{G})$ Nacella magellanica $(27,88 \mathrm{~mm}), \mathrm{H}-\mathrm{I})$ Scurria ceciliana $(5,39$ $\mathbf{m m}$ ) / Gastropoda from Buque Quemado Beach. A-B) Fissurella picta picta (50.63 mm), C- D) Nacella deaurata (42.13 mm), E- G) Nacella magellanica (27.88 mm), H-I) Scurria ceciliana (5.39 mm) 
del género en la costa chilena, concluyendo que todas las especies magallánicas constituyen identidades diferentes con variación intraespecífica (mayormente en la forma, grosor y color de la concha, y morfología de la rádula), a excepción de $N$. fuegiensis (Reeve, 1855), considerada sinónimo de $N$. magellanica. Posteriormente, Aranzamendi et al. (2009), basándose en técnicas moleculares, desarrollaron la identificación de diversas especies del género en el Atlántico Sur y Tierra del Fuego, concluyendo que los especímenes de $N$. delicatissima se presentaron combinados con los especímenes de $N$. magellanica y $N$. deaurata, sugiriendo que $N$. delicatissima es más bien un morfotipo infrecuente de cada una de esas dos especies. Finalmente, GonzálezWevar et al. (2010) estudiaron la filogenia molecular y biogeografía del género en el Océano Austral. $N$. deaurata se distribuye entre la isla de Chiloé y el Cabo de Hornos, alcanzando las Islas Malvinas / Falkland, registrando una batimetría de hasta $30 \mathrm{~m}$ de profundidad (Valdovinos \& Rüth 2005). N. magellanica, por su parte, se ha registrado hasta los $285 \mathrm{~m}$ de profundidad (USNM 2010) y su distribución comprende desde la Isla de Chiloé y el Cabo de Hornos, Islas Malvinas / Falkland (Valdovinos \& Rüth 2005), alcanzando la Provincia de Río Negro, 42 $S$ (Aranzamendi et al. 2009) y aguas bonaerenses (Castellanos \& Landoni 1988). No obstante, $N$. magellanica, también se encuentra registrada en localidades antárticas (Islas Shetland del Sur y Península) y las Islas Antípodas (USNM 2010), registros que necesitan confirmación a través de una comparación con ejemplares de las otras especies del género presentes en esos lugares [i.e., Nacella polaris sensu lato y Nacella terroris (Filhol, 1880), respectivamente].

Scurria ceciliana (Fig. 4H-I) es un patelogastrópodo típico de la costa del Pacifico, distribuyéndose desde el Perú $\left(5^{\circ} \mathrm{S}\right)$ hasta Isla Navarino (Espoz et al. 2004). Esta especie se presenta en intermareales rocosos, ya sea expuestos o protegidos, en plataformas verticales y horizontales y en pozas intermareales (Espoz et al. 2004). Se caracteriza por presentar un ecofenotipo que habita entre los cirripedios de la franja intermareal alta, presentando altos niveles de infección por parte del liquen Thelidium litorale (Espoz et al. 1995), lo cual le da una variabilidad morfológica.

Margarella expansa (Fig. 5A-B) es una especie con una amplio rango batimétrico, encontrándose desde los 0 a los 265 m (USNM 2010). Externamente es muy difícil distinguir entre las formas de coloración más rosadas de $M$. expansa y la conocida especie $M$. violacea (King \& Broderip, 1832). Powell (1951) comentó que $M$. expansa se diferencia de $M$. violacea porque presenta un color más oliváceo y una apertura oval más larga. Más tarde, Zelaya (2004) describió que la concha de $M$. expansa está compuesta de dos capas bien diferenciadas, siendo la capa interna más delgada (Fig. 5B). Se distribuye desde el cañón del Río de la Plata hasta el Estrecho de Le Maire (USNM 2010), incluyendo también las Islas Malvinas / Falkland, Georgias del Sur, el Estrecho de Magallanes (Sowerby 1838), Ushuaia y Tierra del Fuego (Zelaya 2004). Tomando en cuenta la citada similitud que se tiene entre esta especie y M. violacea (e.g., Powell 1951, Smith 1879, Troncoso et al. 2001) y el escaso número de registros de M. expansa dentro de la Provincia Magallánica, sería necesario realizar una revisión de ejemplares desde lo más al norte que se ha registrado $M$. violacea en el Océano Pacífico, i.e., $46^{\circ} \mathrm{S}$ (ver Reid \& Osorio 2000), a fin de poder dilucidar la coexistencia de ambas especies en los diversos ambientes bentónicos.

Trochita pileus (Fig. 5C-D) es una especie muy similar morfológicamente a la especie T. pileolus (d’Orbigny, 1841); pero la diferencia externa más conspicua entre ambas es que T. pileus presenta una protoconcha más lisa, mientras que $T$. pileolus tiene una protoconcha rugosa (ver Linse 2002). Su distribución abarca registros en la Isla Santa María (Aldea \& Valdovinos 2005), hasta el Cabo de Hornos y las Islas Malvinas (Linse 2002). Esta especie presenta un amplio rango batimétrico desde los $0 \mathrm{~m}$ (Aldea \& Valdovinos 2005) hasta los $484 \mathrm{~m}$ (Linse op. cit.).

Pareuthria plumbea (Fig. 5E) es un típico gasterópodo intermareal que se encuentra debajo de las rocas y en piscinas intermareales (Pastorino \& Penchaszadeh 2002, obs. pers.). Esta especie se distribuye en el Océano Atlántico desde la Provincia de Chubut hasta Tierra del Fuego (Castellanos \& Landoni 1992) e Islas Malvinas (Dell 1971), y en el Océano Pacifico desde Puerto Edén hasta el Cabo de Hornos (Dell op. cit.). Se caracteriza porque, a diferencia de otras especies de la familia Buccinidae, presenta dentro de su ciclo reproductivo un desarrollo directo depositando una masa de huevos donde se desarrollan los embriones (Pastorino \& Penchaszadeh op. cit.).

El pulmonado Kerguelenella lateralis (Fig. 5F-G) se caracteriza por ser una especie marcadamente asimétrica, presentando el umbo ubicado posteriormente en el lado izquierdo de la concha, que la hace diferenciarse de todas las variaciones morfológicas de Siphonaria lessoni (ver más abajo). Se distribuye entre el Estrecho de Magallanes y Cabo de Hornos (Powell 1951, Dell 1971), alcanzando por el Atlántico Puerto Deseado ( $\left.48^{\circ} \mathrm{S}\right)$, las Islas Malvinas / Falkland (Castellanos et al. 1993) y Georgias del Sur (Zelaya 


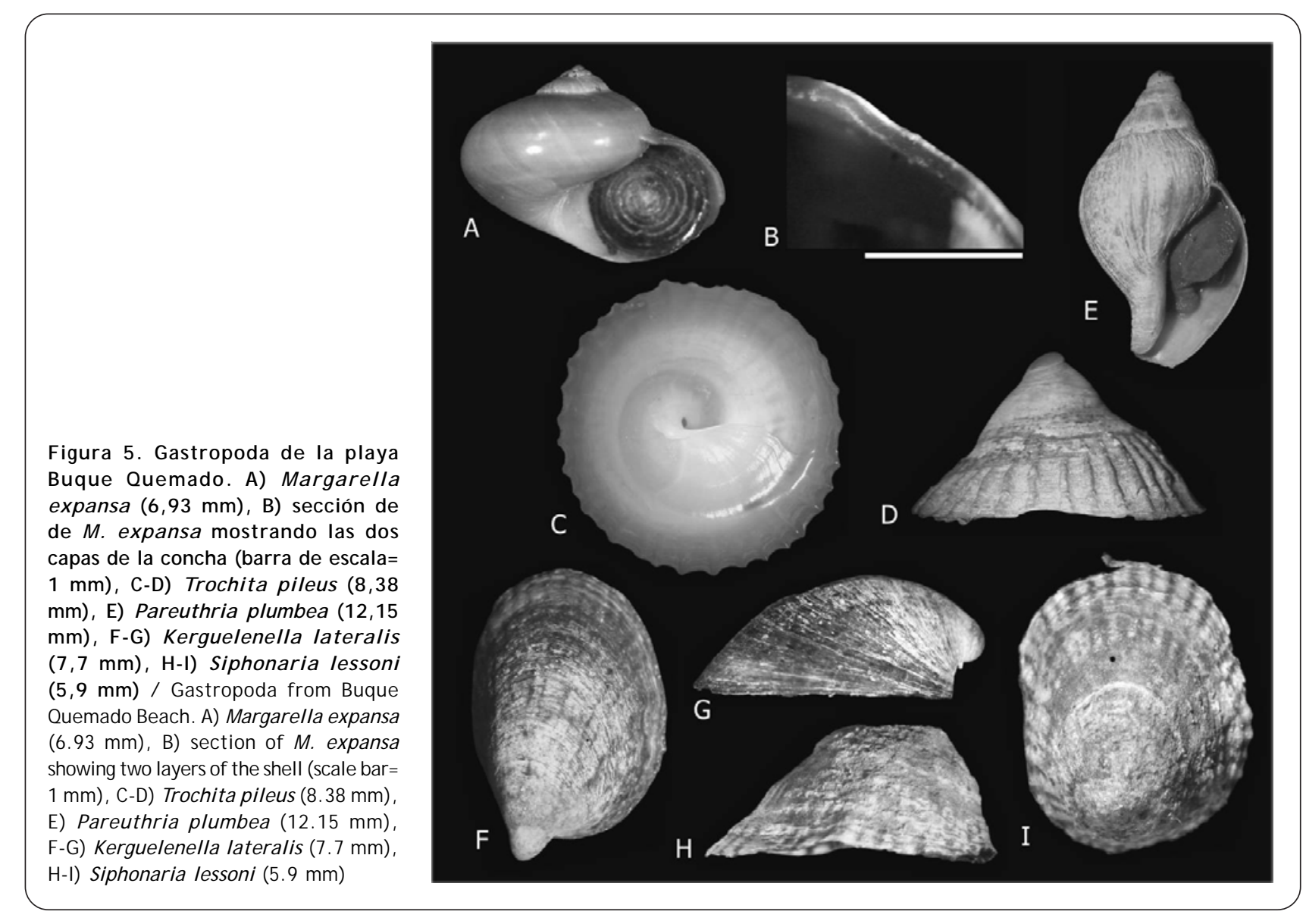

2005). Habita principalmente ambientes intermareales, encontrándose hasta los $5 \mathrm{~m}$ de profundidad (Zelaya 2005). Castellanos et al. (1993) la citan además para Nueva Zelanda, las Islas Kerguelen, Campbell y Antípodas, pero Powell (1951) había comentado que dichos registros correspondían a una especie no descrita.

Siphonaria lessoni (Fig. 5H-I) es una especie común netamente intermareal, que presenta una concha variable. Al respecto, Tablado \& López-Gappa (2001) estudiaron la diversidad morfométrica en distintos ambientes costeros en un sector de la costa atlántica (Provincia de Buenos Aires) en que habita la especie, demostrando una alta variabilidad influenciada por las características físicas y ambientales del hábitat. Presenta una amplia distribución, registrándose en el Pacífico desde Paita en Perú (Alamo \& Valdivieso 1997) hasta el Cabo de Hornos (Dell 1971) y, en el Atlántico abarcando las Islas Malvinas y la costa patagónica (Castellanos et al. 1993) hasta Uruguay (Scarabino 2004) y la costa de Santa Catarina en Brasil (Penchaszadeh et al. 2007); aunque también está citada para las Islas Kerguelen
(Powell 1951) y Sudáfrica (Castellanos et al. 1993).

El bivalvo Mytilus edulis chilensis (Fig. 6A-B) es una subespecie bastante común y estudiada, que además constituye una importante pesquería en la costa de Chile (Osorio 2002). Diversos estudios se han desarrollado en torno a su relación con la especie M. edulis Linnaeus, 1758 (ver Reid \& Osorio 2000, p. 131), no encontrándose aún una respuesta concluyente al tema. Al respecto, Toro (1998), basándose en estudios genéticos y morfológicos, propuso su estado taxonómico como M. edulis chilensis, criterio que ha sido más ampliamente utilizado en diversos reportes e investigaciones y que se utilizó en este trabajo, esperando que las relaciones geográficas de la especie $M$. edulis y sus potenciales subespecies estén completamente resueltas. Sin embargo, Cárcamo et al. (2005) realizaron un estudio sobre ejemplares de la costa de Chile, en base a aloenzimas, comparando los resultados con ejemplares europeos de $M$. edulis y M. galloprovincialis Lamarck, 1819, concluyendo que el estado taxonómico de los ejemplares chilenos podría ser M. galloprovincialis chilensis, debido a que 


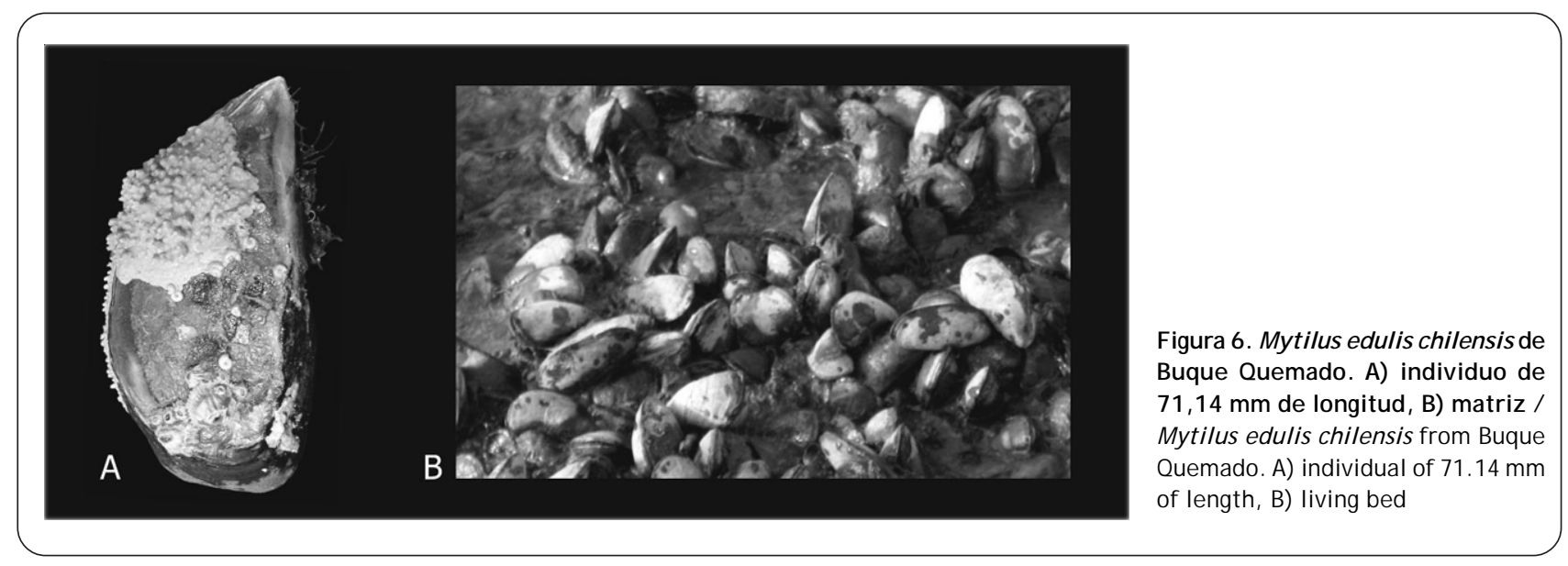

genéticamente estuvieron más cercanas a la segunda especie, pero teniendo frecuencias de alelos características y particulares. M. edulis chilensis se distribuye entre $20^{\circ} \mathrm{S}$ y $54^{\circ} \mathrm{S}$ en el Pacífico Sur-oriental, desde el intermareal a $25 \mathrm{~m}$ de profundidad (Reid \& Osorio 2000), registrándose además hasta la Isla Navarino (Ojeda et al. 2010), Isla de los Estados y el Margen oriental de la isla de Tierra del Fuego (USNM 2010). Los registros más al norte (Iquique, $20^{\circ} \mathrm{S}$ ), provienen del reporte de SootRyen (1959), sin embargo, llama la atención la ausencia de registros posteriores en reportes exhaustivos de localidades próximas (e.g., Marincovich 1973, Guzmán et al. 1998), lo cual sugiere un estatus de 'poco común' de la subespecie en el norte de Chile. En el mismo sentido, podría comprobarse la extensión de su distribución hacia el Océano Atlántico, mediante un estudio comparativo con la subespecie M. edulis platensis d'Orbigny, 1842, dado los registros de $M$. edulis chilensis en la costa Atlántica (ver USNM 2010).

El bajo número de especies obtenidas podría responder mayormente a factores físicos de la playa, donde, a pesar de la gran amplitud de la zona intermareal, dominan las grandes extensiones de sustrato blando, presentando aislados parches de roca o bolones pequeños, en donde se concentraron los moluscos. En este sentido, los patrones biológicos se explican por los parámetros ambientales tales como profundidad del agua, presencia de sedimentos blandos y escombros biogénicos (Gutt et al. 1999). Bajos índices de diversidad y riqueza han sido reportados en algunos sitios del Estrecho de Magallanes (Benedetti-Cecchi 1996). Mutschke et al. (1998) registraron un total de 26 especies de moluscos en el intermareal de bloques y cantos de Bahía Laredo (2 Polyplacophora, 20 Gastropoda y 4 Bivalvia) a partir de muestras recolectadas durante varios años de investigación. Posteriormente, Ríos \& Mutschke (1999) registraron 17 especies de moluscos (2 Polyplacophora, 11 Gastropoda y 4 Bivalvia) en 4 transectos realizados en el canal Whiteside, no encontrando macroorganismos en la parte superior arenosa de la playa. El mayor número de especies encontradas en estudios sublitorales en áreas próximas (i.e., 69 especies; Ríos et al. 2003) sugiere considerar los sustratos blandos como un importante factor espacial limitante de la diversidad intermareal, pero además se debe tener en cuenta el factor temporal de un estudio que permita definir la variación de la diversidad en diferentes períodos.

\section{Agradecimientos}

Los autores expresan su gratitud a Johanna Marambio por su colaboración en los trabajos en terreno, al profesor Andrés Mansilla (Universidad de Magallanes e Instituto de Ecología y Biodiversidad) por el uso del laboratorio de Macroalgas de la Universidad de Magallanes y a Carmen Espoz (Universidad Santo Tomás) por su gentil colaboración en la identificación de la especie Scurria ceciliana. Finalmente, se agradece a dos revisores anónimos del manuscrito por sus valiosos comentarios y aportaciones.

\section{LITERATURA CITADA}

Alamo V \& V Valdivieso. 1997. Lista sistemática de moluscos marinos del Perú. Segunda edición, revisada y actualizada, 183 pp. Instituto del Mar del Perú, Callao.

Aldea C \& C Valdovinos. 2005. Moluscos del intermareal rocoso del centro-sur de Chile $\left(36^{\circ}-38^{\circ} \mathrm{S}\right)$ : taxonomía y clave de identificación. Gayana 69(2): 364-396.

Aranzamendi MC, CN Gardenal, JP Martin \& R Bastida. 2009. Limpets of the genus Nacella (Patellogastropoda) from the Southwestern Atlantic: species identification 
based on molecular data. Journal of Molluscan Studies 75: 241-251.

Benedetti-Cecchi L. 1996. Distribution of macroalgae and invertebrate grazers in the Magellan Strait. Berichte zur Polarforschung 190: 70-71.

Benedetti-Cecchi L \& F Cinelli. 1997. Spatial distribution of algae and invertebrates in the rocky intertidal zone of the Strait of Magellan: are patterns general? Polar Biology 18: 337-343.

Brattström H \& A Johanssen. 1983. Ecological and regional zoogeography of the marine benthic fauna of Chile. Sarsia 68: 289-339.

Camus PA. 2001. Biogeografía marina de Chile. Revista Chilena de Historia Natural 74: 587-617.

Cárcamo C, AS Comesaña, FM Winkler \& A Sanjuán. 2005. Allozyme identification of mussels (Bivalvia: Mytilus) on the Pacific coast of South America. Journal of Shellfish Research 24(4): 1101-1115.

Castellanos ZA \& NA Landoni. 1988. Catálogo descriptivo de la malacofauna marina Magallánica 2. Archigastropoda, 40 pp. Comisión de Investigaciones Científicas, Buenos Aires.

Castellanos ZA \& NA Landoni. 1992. Catálogo descriptivo de la malacofauna marina Magallánica 10. Neogatropoda, 43 pp. Comisión de Investigaciones Científicas, Buenos Aires.

Castellanos ZA, NA Landoni \& JR Dadon. 1993. Catálogo descriptivo de la malacofauna marina Magallánica 12. Opistobranchia, 38 pp. Comisión de Investigaciones Científicas, Buenos Aires.

Dell RK. 1964. Antarctic and subantarctic Mollusca: Amphineura, Scaphopoda and Bivalvia. Discovery Reports 33: 93-250.

Dell RK. 1971. The marine mollusca of the Royal Society Expedition to Southern Chile, 1958-59. Records of the Dominion Museum 7(17): 155-233.

Espoz C, G Guzmán \& JC Castilla. 1995. The lichen Thelidium litorale on shells of intertidal limpets: a case of lichen-mediated cryptic mimicry. Marine Ecology Progress Series 119: 191-197.

Espoz C, DR Lindberg, JC Castilla \& WB Simison. 2004. Los patelogastrópodos intermareales de Chile y Perú. Revista Chilena de Historia Natural 77: 257-283.

González-Wevar C, T Nakano, JI Cañete \& E Poulin. 2010. Molecular phylogeny and historical biogeography of Nacella (Patellogastropoda: Nacellidae) in the Southern Ocean. Molecular Phylogenetics and Evolution 56: 115-124.

Gutt J, E Helsen, W Arntz \& A Buschmann. 1999. Biodiversity and community structure of the megaepibenthos in the Magellan region (South America). Scientia Marina 63(Supl. 1): 155-170.

Guzmán N, S Saá \& L Ortlieb. 1998. Catálogo descriptivo de los moluscos litorales (Gastropoda y Pelecypoda) de la zona de Antofagasta, $23^{\circ} \mathrm{S}$ (Chile). Estudios Oceanológicos 17: $17-86$.

Marcus E. 1959. Reports of the Lund University Chile Expedition 1948-49. 36. Lamellariacea und Opisthobranchia. Lunds Universitets Årsskrift (NF) 55: 1-135.

Marincovich LJR. 1973. Intertidal mollusks of Iquique, Chile. Natural History Museum, Los Angeles County Science Bulletin 16: 1-49.

McLean JH. 1984. Systematics of Fissurella in the Peruvian and Magellanic faunal provinces (Gastropoda: Prosobranchia). Contributions in Science, Natural History Museum of Los Angeles County 354: 1-70.

Mutschke E, C Ríos \& A Montiel. 1998. Situación actual de la macrofauna presente en el intermareal de bloques y cantos de Bahía Laredo, Estrecho de Magallanes. Anales del Instituto de la Patagonia, Serie Ciencias Naturales 26: 5-29.

Linse K. 1997. Die Verbreitung epibenthischer Mollusken im chilenischen Beagle-Kanal. Berichte zur Polarforschung 228: 1-131.

Linse K. 2002. The shelled Magellanic Mollusca: with special reference to biogeography relations in the Southern Ocean, Theses Zoologicae 34: 1-252. A.R.G. Gantner Verlag KG, Ruggell, Lichtenstein.

Ojeda JA, T Contador, S Rosenfeld, CB Anderson, A Mansilla \& JH Kennedy. 2010. Guía para la identificación de los invertebrados marinos y dulceacuícolas de la Reserva de Biósfera Cabo de Hornos, 80 pp. Ediciones Universidad de Magallanes, Punta Arenas.

Osorio C. 2002. Moluscos marinos en Chile, especies de importancia económica, 211 pp. Facultad de Ciencias, Universidad de Chile, Santiago.

Palma S \& N Silva. 2006. Producción científica del Programa CIMAR en los canales y fiordos australes. Cruceros CIMAR 1 a 4 Fiordos, pp. 145-162. Comité Oceanográfico Nacional, Pontificia Universidad Católica de Valparaíso, Valparaíso.

Pastorino G \& PE Penchaszadeh. 2002. Spawn of the Patagonian gastropod Pareuthria plumbea (Philippi, 1844) (Buccinidae). The Nautilus 116(3): 105-108.

Penchaszadeh P, G Pastorino \& M Brögger. 2007. Moluscos gasterópodos y bivalvos: Siphonaria lessoni. En: Boltovskoy D (ed). Atlas de sensibilidad ambiental de la costa y el Mar Argentino. [en línea] <http://atlas.ambiente.gov.ar/>.

Powell AWB. 1951. Antarctic and Subantarctic mollusca: Pelecypoda and Gastropoda. Discovery Reports 26: 47196.

Powell AWB. 1973. The patellid limpets of the world (Patellidae). Indo-Pacific Mollusca 3: 75-206.

Reid DG \& C Osorio. 2000. The shallow-water marine mollusca of the Estero Elefantes and Laguna San Rafael, southern Chile. Bulletin of the Natural History Museum of London, Zoology 66(2): 109-146. 
Ríos C \& D Gerdes. 1997. Ensamble bentónico epifaunístico de un campo intermareal de bloques y cantos en Bahía Laredo, Estrecho de Magallanes. Anales del Instituto de la Patagonia, Serie Ciencias Naturales 25: 47-55.

Ríos C \& E Mutschke. 1999. Community structure of intertidal boulder-cobble fields in the Straits of Magellan, Chile. Scientia Marina 63(Supl. 1): 193-201.

Ríos C, E Mutschke \& E Morrison. 2003. Biodiversidad bentónica sublitoral en el estrecho de Magallanes, Chile. Revista de Biología Marina y Oceanografía 38(1): 1-12.

Scarabino F. 2004. Lista sistemática de los Gastropoda marinos y estuarinos vivientes de Uruguay. Comunicaciones de la Sociedad Malacológica del Uruguay 8(84-85/86-87): 305-346.

Schwabe E, G Försterra, V Häussermann, RR Melzer \& M Schrödl. 2006. Chitons (Mollusca: Polyplacophora) from the southern Chilean Comau Fjord, with reinstatement of Tonicia calbucensis Plate, 1897. Zootaxa 1341: $1-27$.

Sirenko B. 2006. Report on the present state of our knowledge with regard to the chitons (Mollusca: Polyplacophora) of the Magellan Strait and Falkland Islands. Venus 65(1-2): 81-89. [Japanese Journal of Malacology]

Smith EA. 1879. Mollusca. An account of the petrological, botanical and zoological collection made in Kerguelen's Island and Rodriguez during the Transit of 'Venus' Expedition in the years 1874-75. Philosophical Transactions of the Royal Society of London 168: 167192.

Soot-Ryen T. 1959. Reports of the Lund University Chile Expedition 1948-49.35. Pelecypoda. Lunds Universitets Årsskrift (NF) 55(6): 1-86.

Sowerby GBI. 1838. A descriptive catalogue of the species of Leach's genus Margarita. Malacological and Conchological Magazine 1: 23-27.

Strebel H. 1904. Beiträge zur Kenntnis der Molluskenfauna der Magalhaen-Provinz. Zoologische Jahrbücher. Abteilung für Systematik Geographie und Biologie der Tiere 21: 171-248.

Strebel H. 1905a. Beiträge zur Kenntnis der Molluskenfauna der Magalhaen-Provinz, II. Die Trochiden. Zoologische Jahrbücher Suppl. 8: 121-166.
Strebel H. 1905b. Beiträge zur Kenntnis der Molluskenfauna der Magalhaen-Provinz. No 3. Zoologische Jahrbücher. Abteilung für Systematik, Geographie und Biologie der Tiere 22: 575-666.

Strebel H. 1906. Beiträge zur Kenntnis der Molluskenfauna der Magalhaen-Provinz. No 4. Zoologische Jahrbücher. Abteilung für Systematik, Geographie und Biologie der Tiere 24: 91-174.

Strebel H. 1907. Beiträge zur Kenntnis der Molluskenfauna der Magalhaen-Provinz. No 5. Zoologische Jahrbücher. Abteilung für Systematik, Geographie und Biologie der Tiere 25: 79-196.

Stuardo J. 1964. Distribución de los moluscos marinos litorales en Latinoamérica. Boletín del Instituto de Biología Marina 7: 79-91.

Tablado A \& J López-Gappa. 2001. Morphometric diversity of the pulmonate limpet Siphonaria lessoni in different coastal environments. Scientia Marina 65(1): 33-41.

Toro J. 1998. PCR-based nuclear and mtDNA markers and shell morphology as an approach to study the taxonomy status of the Chilean blue mussel, Mytilus chilensis (Bivalvia). Aquatic Living Resources 11(5): 347-353.

Troncoso N, JL Van Goethem \& JS Troncoso. 2001. Contribution to the marine molluscan fauna of Kerguelen Island, south Indian ocean. Iberus 19(1): 83-114.

USNM. 2010. National Collection of the Smithsonian National Museum of Natural History, U.S. National Collection. [en línea] <http://invertebrates.si.edu/>.

Valdenegro A \& N Silva. 2003. Caracterización oceanográfica física y química de la zona de canales y fiordos australes de Chile entre el Estrecho de Magallanes y Cabo de Hornos (CIMAR 3 Fiordos). Ciencia y Tecnología del Mar 26(2): 19-60.

Valdovinos C \& M Rüth. 2005. Nacellidae limpets of the southern end of South America: taxonomy and distribution. Revista Chilena de Historia Natural 78: 497-517.

Zelaya DG. 2004. The genus Margarella Thiele, 1893 (Gastropoda: Trochidae) in the southwestern Atlantic ocean. The Nautilus 118(3): 112-120.

Zelaya DG. 2005. Systematics and biogeography of marine gastropod molluscs from South Georgia. Spixiana 28(2): 109-139.

Recibido el 06 de enero 2011 y aceptado el 12 de febrero 2011 